\title{
Qualitative and Quantitative Comparison of Liquid-Liquid Phase Extraction Using Ethyl Acetate and Liquid-Solid Phase Extraction Using Poly-Benzyl-Resin for Natural Products
}

\author{
Yannik K. Schneider*(D), Solveig M. Jørgensen, Jeanette Hammer Andersen (D) and Espen H. Hansen (D) \\ Marbio, Faculty for Fisheries, Biosciences and Economy, UiT-The Arctic University of Norway, Breivika, \\ N-9037 Tromsø, Norway; solveimj@stud.ntnu.no (S.M.J.); jeanette.h.andersen@uit.no (J.H.A.); \\ espen.hansen@uit.no (E.H.H.) \\ * Correspondence: yannik.k.schneider@uit.no; Tel.: +47-(0)-77649267
}

Citation: Schneider, Y.K.; Jørgensen, S.M.; Andersen, J.H.; Hansen, E.H. Qualitative and Quantitative Comparison of Liquid-Liquid Phase Extraction Using Ethyl Acetate and Liquid-Solid Phase Extraction Using Poly-Benzyl-Resin for Natural Products. Appl. Sci. 2021, 11, 10241. https://doi.org/10.3390/ app112110241

Academic Editors: Guillaume Pierre and Emanuel Vamanu

Received: 31 August 2021

Accepted: 29 October 2021

Published: 1 November 2021

Publisher's Note: MDPI stays neutral with regard to jurisdictional claims in published maps and institutional affiliations.

Copyright: (c) 2021 by the authors. Licensee MDPI, Basel, Switzerland. This article is an open access article distributed under the terms and conditions of the Creative Commons Attribution (CC BY) license (https:// creativecommons.org/licenses/by/ $4.0 /)$.

\begin{abstract}
A key step in the process of isolating microbial natural products is the preparation of an extract from a culture. This step determines which molecules will be available for detection in the subsequent chemical and biological analysis of a biodiscovery pipeline. In the present study we wanted to document potential differences in performance between liquid-liquid extraction using ethyl acetate and liquid-solid extraction using a poly-benzyl-resin. For the comparison of the two extraction protocols, we spiked a culture of Flavobacterium sp. with a diverse selection of natural products of microbial and plant origin to investigate whether the methods were comparable with respect to selectivity. We also investigated the efficiency of the two extraction methods quantitatively, using water spiked with a selection of natural products, and studied the quantitative effect of different $\mathrm{pH}$ levels of the aqueous solutions on the extraction yields of the two methods. The same compounds were extracted by the two methods, but the solid-phase extract contained more media components compared with the liquid-phase extract. Quantitatively, the two extraction methods varied in their recovery rates. We conclude that practical aspects could be more important when selecting one of the extraction protocols, as their efficiencies in extracting specific compounds were quite similar.
\end{abstract}

Keywords: natural products; bacteria; downstream processing; antibiotics; isolation; extraction; secondary metabolites; pharmacognosy; bioprospecting

\section{Introduction}

Natural products and their derivatives have successfully been developed as medicines that have enabled the treatment of various diseases [1]. Natural products differ from compounds that can be found in synthetic screening libraries by occupying a different chemical space that makes them a valuable source for new chemical scaffolds [2]. Microorganisms in general and bacteria in particular are promising sources for new bioactive natural products that can be developed as drug leads [3,4]. Natural product drug discovery commonly starts with the preparation of an extract, and this extract maybe pre-fractionated or directly investigated for bioactivity using bioassays such as anti-microbial or anti-cancer assays [5,6]. If bioactivity is detected, extracts or fractions are subsequently investigated for the presence of known active metabolites, which will then be removed from the pipeline [7]. This process is called dereplication and usually relies on hyphenated mass spectrometry techniques such as HPLC-MS [8,9]. An extract may also be investigated for novel metabolites without preceding bioactivity testing [10]. Potential new compounds will be isolated and subjected to structural elucidation and bioactivity profiling.

Extraction is a crucial step in the search for new active compounds. If a compound is not successfully extracted from the biomass or fermentation broth, it can obviously not be detected in downstream bioassays or chemical analysis. For bacterial fermentations, many possible extraction techniques and protocols exist, but as it is usually not feasible 
to apply more than one of them when screening larger collections of bacterial isolates, it is important to be aware of the limitations of the selected protocol. While we have used liquid-solid phase extraction of fermentation broths in previous studies [11,12], there are other relevant techniques such as liquid-liquid partition with ethyl acetate (EtOAc) [13] or extraction of the bacterial mycelium with ethanol in case of actinobacteria [14]. Sample pre-treatment and solvent selection are also dependent on whether intracellular or secreted metabolites are of interest [15].

To assess the suitability of our standard method for extraction of bacterial and fungal fermentations (liquid-solid phase extraction), we conducted a small study to compare it with liquid-liquid partition using EtOAc. We investigated the qualitative difference when extracting a bacterial culture spiked with seven different natural products. Socalled spike and recovery tests are an established method for verifying the suitability and detection limits of an analytical method or sample preparation, including extraction procedures [16,17].

In addition, the two methods were compared quantitatively by extraction of four of the natural products from aqueous solution. We also investigated a potential effect of the $\mathrm{pH}$ level of the aqueous solution on the extraction yields.

\section{Materials and Methods}

The chemicals used were of appropriate purity, as indicated by the supplier, and pure water $\left(\mathrm{pH}_{2} \mathrm{O}\right)$ was produced using an in-house MilliQ system. For vacuum filtrations, a Büchner funnel with Whatman No. 3 filter paper (Whatman plc., Maidstone, UK) was used.

\subsection{Preparation of the Spiked Culture}

A Flavobacteria sp. isolate was cultured over $7 \mathrm{~d}$ at $10^{\circ} \mathrm{C}$ in a $450 \mathrm{~mL}$ shaking bottle culture, shaking at $300 \mathrm{rpm}$. A DVR1 medium was used for cultivation, consisting of $6.0 \mathrm{~g}$ malt extract (Sigma-Aldrich, St. Louis, MO, USA), $10.0 \mathrm{~g}$ Peptone from casein (Sigma-Aldrich), $6.0 \mathrm{~g}$ yeast extract (Sigma-Aldrich), $450 \mathrm{~mL}$ filtrated seawater and $450 \mathrm{~mL}$ $\mathrm{pH}_{2} \mathrm{O}$ [11]. The media was sterilized by autoclaving at $120^{\circ} \mathrm{C}$ for $30 \mathrm{~min}$ under pressure and inoculated using an inoculation loop. After cultivation, the fermentation broth was centrifuged at $4000 \mathrm{rpm}$ for $10 \mathrm{~min}$ to remove the cells using a Multifuge $3 \mathrm{~S}-\mathrm{R}$ equipped with a SORVALL 75,006,445 rotor (Heraeus GmbH \& Co. KG, Hanau, Germany).

An aliquot of $300 \mathrm{~mL}$ of the supernatant was spiked with eight natural productsnamely, rifampicin (R3501, Sigma-Aldrich), vancomycin (Sigma-Aldrich), colchicine (C3915, Sigma-Aldrich), cyclosporine (32425, Fluka ${ }^{\circledR}$, Honeywell, Charlotte, NC, USA), paclitaxel (86346, Fluka ${ }^{\circledR}$, Honeywell), ampicillin (A5354, Sigma-Aldrich) and gentamycin (A2712, Merck, Darmstadt, Germany). The concentrations of the respective natural products are given in Table 1 (structures in Figure 1). After the supernatant was spiked, it was thoroughly mixed and split into two samples of $150 \mathrm{~mL}$ each. The two samples were processed in parallel; one was extracted using liquid-liquid extraction and one using liquid-solid extraction.

Table 1. Final concentrations of the natural products in the bacterial culture.

\begin{tabular}{ccc}
\hline Compound & Natural Product Class & Concentration $(\mu \mathrm{g} / \mathrm{L})$ \\
\hline Rifampicin $^{*}$ & polyketide & 2.0 \\
Vancomycin $^{\dagger}$ & glycopeptide & 10.0 \\
Colchicine $^{+}$ & alkaloid & 5.0 \\
Tetracycline $^{*}$ & polyketide & 10.0 \\
Cyclosporine $^{*}$ & cyclic peptide & 10.0 \\
Paclitaxel $^{*}$ & terpenoide & 2.0 \\
Ampicillin & B-lactam antibiotic & 10.0 \\
Gentamycin $\ddagger$ & aminoglycoside & 10.0 \\
\hline
\end{tabular}

* dissolved in ethanol; ${ }^{\dagger}$ dissolved in $\mathrm{pH}_{2} \mathrm{O} ;{ }^{\ddagger}$ readymade solution. 
<smiles>COc1cc2c(c(OC)c1OC)-c1ccc(OC)c(=O)cc1[C@@H](NC(C)=O)CC2</smiles>

1

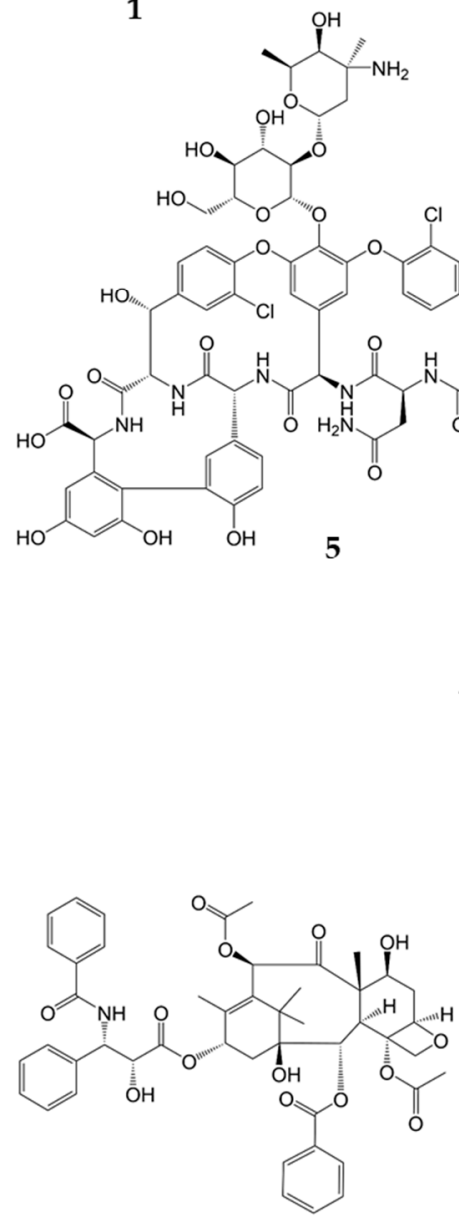

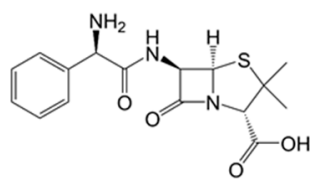

2

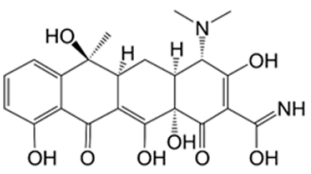

3<smiles>CNC(C)C1CCC(N)C(OC2C(O)C(OC3OCC(C)(O)C(NC)C3O)C(N)C[C@H]2N)O1</smiles>

Figure 1. Structures of the natural products used for spiking within this study. (1) Colchicine; (2) Ampicillin; (3) Tetracycline; (4) Gentamycin; (5) Vancomycin; (6) Cyclosporine A; (7) Paclitaxel;

(8) Rifampicin.

\subsection{Extraction}

\subsubsection{Liquid-Liquid Phase Extraction}

For the liquid-liquid phase extraction, the $150 \mathrm{~mL}$ sample was extracted three times using $75 \mathrm{~mL}$ EtOAc (Sigma-Aldrich) and a separation funnel. The extracts were reduced to dryness at $40{ }^{\circ} \mathrm{C}$ under reduced pressure, and the yield was determined gravimetrically.

\subsubsection{Liquid-Solid Phase Extraction}

For the liquid-solid phase extraction, Diaion ${ }^{\circledR}$ HP-20 (SUPELCO, Sigma-Aldrich) resin was used. An amount of $6.75 \mathrm{~g}$ resin was soaked in methanol (MeOH, HiPerSolv, VWR, Radnor, PA, USA) for $30 \mathrm{~min}$ for activation and transferred to $\mathrm{pH}_{2} \mathrm{O}$ to wash out $\mathrm{MeOH}$ ( $\sim 4.5 \mathrm{~g}$ resin was used for $100 \mathrm{~mL}$ sample or culture). After $20 \mathrm{~min}$ of washing in $\mathrm{pH}_{2} \mathrm{O}$, the resin was added to the spiked fermentation broth and shaken for $72 \mathrm{~h}$ at $10^{\circ} \mathrm{C}$ for extraction. The resin was filtered from the sample using a cheese-cloth filter (1057, Dansk Hjemmeproduktion, Ejstrupholm, Denmark) placed on a Büchner funnel and subsequently washed in $\mathrm{pH}_{2} \mathrm{O}$. Thereafter the resin was eluted twice using $50 \mathrm{~mL} \mathrm{MeOH}$. Extraction was done by eluting the resin in $\mathrm{MeOH}$ for $1 \mathrm{~h}$ and by subsequently removing the resin by 
vacuum filtration. The extracts were reduced to dryness at $40{ }^{\circ} \mathrm{C}$ under reduced pressure, and the yield was determined gravimetrically.

2.3. Preparation and Extraction of Spiked Water for Quantitative Comparison and Investigation of the $\mathrm{pH}$ Effect on Extraction Yields

Stock solutions of tetracycline $(10 \mathrm{mg} / \mathrm{mL}$ aq.), cyclosporine $(10 \mathrm{mg} / \mathrm{mL}$ in EtOH), colchicine $(5 \mathrm{mg} / \mathrm{mL}$ in $\mathrm{EtOH})$ and gentamicin $(10 \mathrm{mg} / \mathrm{mL}$ aq. $)$ were prepared to spike the five water samples. Five $200 \mathrm{~mL}$ samples of $\mathrm{pH}_{2} \mathrm{O}$ were set to a $\mathrm{pH}$ of 4.0, 5.0, 6.0, 7.0 and 8.5, respectively, using $1 \mathrm{M} \mathrm{HCl}$ (EMSURE, Merck), $0.3 \mathrm{M} \mathrm{NaOH}$ (VWR) and a pH meter (WTW InoLab pH 720, Xylem Inc. Rye Brook, NY, USA) equipped with a SenTix ${ }^{\circledR} 41$ electrode (WTW). The five conditions were spiked with the natural products to reach a final concentration of $10 \mu \mathrm{g} / \mathrm{mL}$ for tetracycline, cyclosporine A and gentamicin and $5 \mu \mathrm{g} / \mathrm{mL}$ for colchicine. Thereafter the five conditions (each $200 \mathrm{~mL}$ ) were split into two aliquots of $100 \mathrm{~mL}$ each for parallel extraction using liquid-liquid phase extraction and liquid-solid phase extraction. For liquid-liquid phase extraction, the $100 \mathrm{~mL}$ aliquots were extracted with $100 \mathrm{~mL}$ EtOAc using a separation funnel. After shaking the two phases for $\sim 1 \mathrm{~min}$, the funnel was left to rest until the two phases separated properly. The EtOAc fractions were reduced to dryness at $40^{\circ} \mathrm{C}$ under reduced pressure. The other aliquot for each water sample for resin or liquid-solid phase extraction was extracted using resin as described above (using $\sim 4.5 \mathrm{~g}$ resin/100 $\mathrm{mL}$ of sample).

\subsection{Analysis of the Extracts}

\subsubsection{Gravimetrical Analysis}

The yields were determined gravimetrically using an analytical balance (Mettler Toledo AB204-S, Mettler Toledo, Columbus, OH, USA).

\subsubsection{UHPLC-IMS-MS Analysis}

For detection of the compounds, the extracts were re-dissolved in DMSO (SigmaAldrich) to a final concentration of $40 \mathrm{mg} / \mathrm{mL}$ and diluted $1: 4$ with $80 \% \mathrm{MeOH}$ aq. to prepare an injection solution for UHPLC analysis.

For analysis of the presence of the respective natural products, UHPLC-IMS-MS was used. The analytical system consisted of an Acquity I-class UPLC (Waters, Milford, MA, USA) coupled to a PDA detector and a Vion IMS QToF (Waters). The chromatographic separation was performed using an Acquity BEH C18 UPLC column $(1.7 \mu \mathrm{m}$, $2.1 \mathrm{~mm} \times 100 \mathrm{~mm}$ ) (Waters). Mobile phases consisted of acetonitrile (HiPerSolv, VWR) for mobile phase $\mathrm{B}$ and $\mathrm{pH}_{2} \mathrm{O}$ produced by the in-house Milli-Q system as mobile phase $\mathrm{A}$, both containing $0.1 \%$ formic acid $(v / v)$ (Merck). The gradient was run linearly from $10 \%$ to $90 \%$ B over $12 \mathrm{~min}$ at a flow rate of $0.45 \mathrm{~mL} / \mathrm{min}$. Samples were run in ESI+ and ESI- ionization mode. The data were processed and analyzed using UNIFI 1.9.4 (Waters). Stock solutions of the respective natural products were injected as reference for unambiguous identification and to confirm that the compounds were detectable within the analytical setup.

For the quantitative analysis, the extracts were dissolved in $1.0 \mathrm{~mL} 80 \% \mathrm{MeOH}$ aq., and a 10-fold dilution with $80 \% \mathrm{MeOH}$ aq. was prepared for injection. A sample of each of the four stock solutions was diluted to $0.1 \mathrm{mg} / \mathrm{mL}$ in $80 \% \mathrm{MeOH}$ aq. to obtain a reference solution. An amount of $5.0 \mu \mathrm{L}$ was injected and analyzed using the same UHPLC protocol as above. For the quantification, three injections per sample were made, and the response/ion count of the respective $\mathrm{m} / \mathrm{z}$ signal of the pseudo-molecular ion was used for quantification. The results were analyzed using GraphPad Prism 8.1.0 for linear regression.

\section{Results}

\subsection{Qualitative Comparison}

The EtOAc extraction of the spiked bacterial ferment was done three times with the same spiked sample, and the yield was determined for each of the three extractions 
individually. The yields for the three extractions were 26.9, 11.7 and $15.0 \mathrm{mg}$, respectively. The combined yield of the extractions was $53.6 \mathrm{mg}$ (dry weight). The liquid-solid extraction yielded $325.7 \mathrm{mg}$ of extract. The first EtOAc extract and the solid-phase extract were analyzed using UHPLC-IMS-MS and investigated for the presence of the spiked natural products. A small quantity of the stock solutions was injected as reference to determine the retention time and mass spectra for the respective natural products. The extracts were investigated for the presence of the spiked compounds by comparison with the retention time and mass spectra of the references. The results for the qualitative comparison of the extraction methods are given in Table 2.

Table 2. Retention times and presence of the natural products in EtOAc and resin extracts.

\begin{tabular}{ccccc}
\hline Compound & $\begin{array}{c}\text { Ret. Time } \\
(\mathbf{m i n})\end{array}$ & Mw & \multicolumn{2}{c}{ Present in Extracts: } \\
EtOAc & Resin \\
\hline Rifampicin & 6.06 & 822.9 & + & + \\
Vancomycin & 0.97 & 1449.2 & + & + \\
Tetracycline & 2.09 & 444.4 & + & + \\
Colchicine & 3.30 & 399.4 & + & + \\
Cyclosporine & 9.60 & 1202.6 & + & + \\
Paclitaxel & 6.51 & 853.9 & + & - \\
Ampicillin & 1.51 & 349.4 & - & - \\
Gentamycin & 0.46 & 482.5 & - & - \\
\hline
\end{tabular}

When comparing the chromatograms from the liquid-liquid and liquid-solid extractions, it appeared that many more polar metabolites and media components were extracted using the liquid-solid phase extraction compared with the liquid-solid phase extraction.

\subsection{Quantitative Comparison}

Water samples with $\mathrm{pH}$ values of 4.0, 5.0, 6.0, 7.0 and 8.5 were spiked with natural products, and an aliquot of each sample was extracted using EtOAc as well as resin extraction, as described above. For the quantitative comparison, the response of the protonated pseudo-molecular ions of the respective standard injections was used as reference to calculate the concentrations of the respective spike compounds. For determination of a calibration curve, LOQ and LOD, we injected 0.05, 0.25, 0.5 and $1 \mu \mathrm{g}$ of cyclosporine, gentamycin, colchicine and tetracycline using the analytical protocol described above. We also injected $0.25 \mu \mathrm{g}$ and $0.05 \mu \mathrm{g}$ of ampicillin to determine the LOD of ampicillin that was not detected in the qualitative study. The calibration curves are shown in Figure 2. The LOD for ampicillin was $<0.05 \mu \mathrm{g}$; LOD for tetracycline, cyclosporine and colchicine was $<0.05 \mu \mathrm{g}$; LOD for gentamycin was $<0.25 \mu \mathrm{g}$, and a proper quantification of gentamycin failed (see Figure 2). We also failed to detect gentamycin at $0.05 \mu \mathrm{g}$ in negative electrospray. The extracts were dissolved in $1.0 \mathrm{~mL}$ of $80 \% \mathrm{MeOH}$ aq., and the final yields were determined by multiplying the concentration with the volume; the final yields are given in Appendix A. Relative yields were calculated as ratios of the initial quantity of spike compound in the $100 \mathrm{~mL}$ of aqueous solution to be extracted. The relative yields are given in Figure 3. 


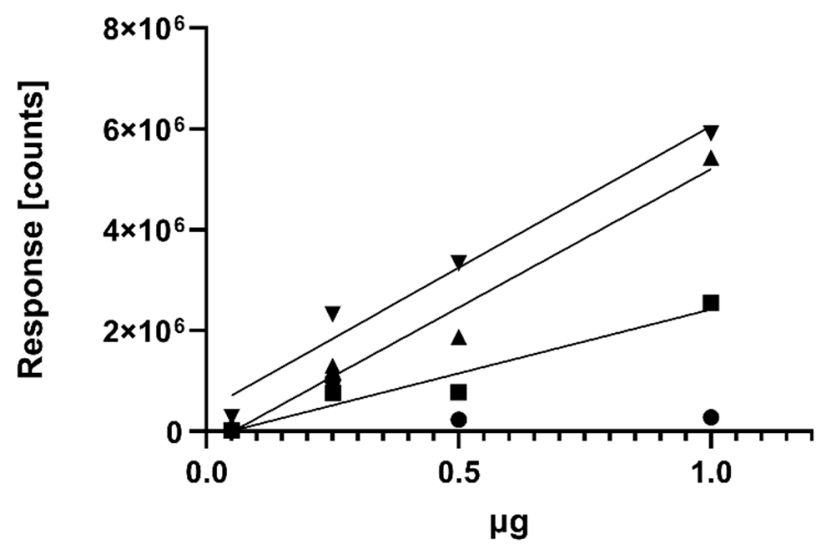

- Gentamycin

- Cyclosporine

- Tetracycline

$\rightarrow$ Colchicine

Figure 2. Calibration curves for the quantification of the selected natural products. Single injections were used for the calibration curves. The formulas and $\mathrm{R}^{2}$ values for the calibration curves were tetracycline $y=5487981 x-112802,0.97$; cyclosporine $253601 x-11282, R^{2}=0.93$; colchicine $y=$ $5617051 x+438251, R^{2}=0.97$; with $y$ being the response and $x$ being the injected compound quantity in $\mu \mathrm{g}$. We failed to generate a proper calibration curve for gentamycin.

\section{A}

EtOAc-/ liquid-liquid phase extract
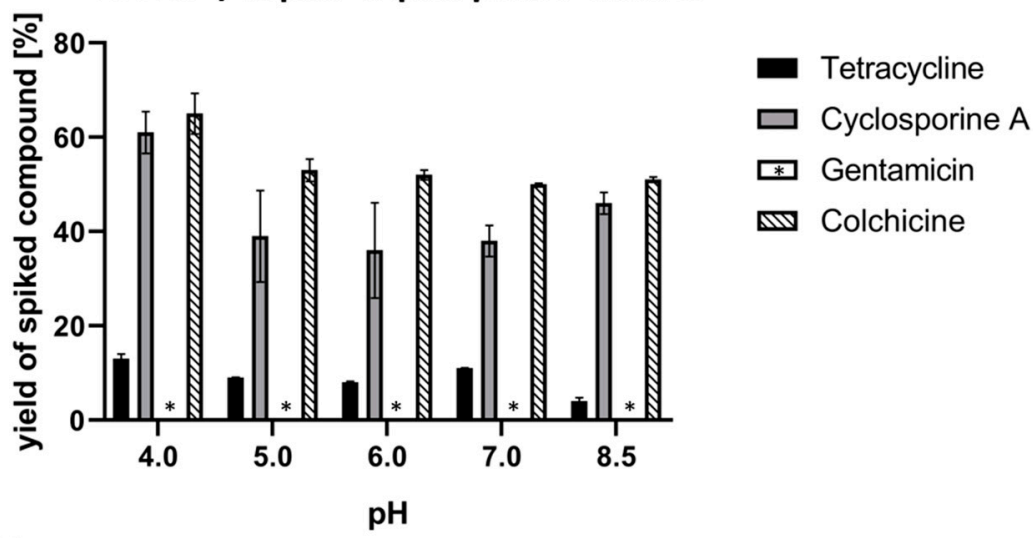

B

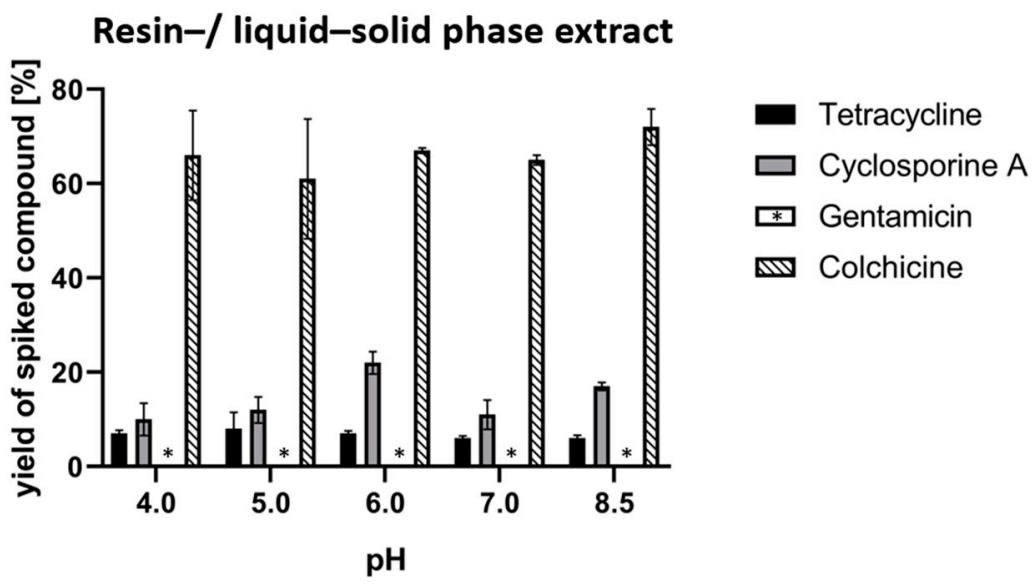

Figure 3. Yield of the natural products in percentage recovery from the spiked solution. A: Results for the liquid-liquid phase extraction. B: Results for the liquid-solid phase extraction. The different $\mathrm{pH}$ levels for the aqueous solutions/extraction conditions are given on the $x$-axis. Note that gentamycin $\left({ }^{*}\right)$ was not detected in any of the two extracts; however, the LOD for gentamycin would be around $5.0 \%$ of the initially spiked quantity; given the extract was dissolved in $1.0 \mathrm{~mL}$ of solvent, for all the other compounds LOD would equal $<1.0 \%$ of the initially spiked compound quantity. 


\section{Discussion}

The number of different protocols that can be used for extraction of microbial secondary metabolites is high, and they range from classical liquid-liquid extractions to more sophisticated applications, such as ultrasound assisted extraction and supercritical fluid extraction $[18,19]$. Furthermore, the selection of extraction solvents will have an impact on extracted metabolites, as observed in plant material [20]. However, we chose the two selected techniques in this investigation because they required no specialized equipment that went beyond the common equipment in a chemical laboratory, and they were widely used. When establishing the biodiscovery pipeline for bioactive secondary metabolites in our laboratory, we were not able to find a direct experimental comparison of the extraction methods using a spike and recovery test. To assess the potential difference of extraction efficacy and to make our data available to others were therefore the main objectives of this study.

\section{Qualitative Comparison of the Extraction Methods}

The first finding of the qualitative comparison of the extraction methods was the difference in yield when extracting the bacterial cultures. The yield of the resin extract was $325.7 \mathrm{mg}$, and this was about 6 times higher compared with the EtOAc extract, yielding $53.6 \mathrm{mg}$. Investigation of the chromatograms of the UHPLC-MS analysis revealed that the resin extract contained a higher share of polar compounds that originated from bacteria and media, as comparison with a media reference for DVR1 media confirmed. For the targeted natural products, the two extraction methods were quite comparable, as we detected the same spiked natural products in the resin and the EtOAc extracts (Table 2). Interestingly, ampicillin and gentamycin were not extracted by any protocols or at least they were not extracted in sufficient quantity for detection. When comparing the retention times in Table 2, it appears that ampicillin and gentamycin had rather short retention times (1.51 and $0.46 \mathrm{~min}$, respectively) and vancomycin eluted between them $(0.97 \mathrm{~min})$. We speculate that both extraction methods are rather unsuitable for very polar and hydrophilic compounds. When comparing the structures of the analytes, it appears that vancomycin ( 5 in Figure 1 ) is a relatively large molecule $(1449.2 \mathrm{u})$ and consists of five benzylic rings, while ampicillin $(2,349.4 \mathrm{u})$ and gentamycin $(4,482.5 \mathrm{u})$ have substantially lower molecular weights.

For the quantitative comparison of the two extraction protocols, water was used as matrix to dissolve the natural products for reproducibility reasons since the media com-ponents and bacterial metabolites in a fermentation could affect the extraction (as we observed above that the solid-phase extract contained significantly more of those compounds). We intended to compare the extraction of the natural products in a simple way, from purified water, including the effect of the $\mathrm{pH}$ levels of the aqueous solutions on the extraction yield. Some bacteria prefer to grow or grow exclusively at acidic (acidophil bacteria) [21] or alkalic $\mathrm{pH}$ levels (alkaliphile bacteria) [22], and we also wanted to investigate if a post-fermentative change in $\mathrm{pH}$ by adding acid or alkali could be a strategy to increase yields. For the quantitative study, we selected four natural products (tetracycline, cyclosporine, colchicine and gentamycin), trying to cover a broad range of polarities and molecular weights. We investigated $\mathrm{pH}$ levels of 4.0, 5.0, 6.0, 7.0 and 8.5. In Figure 3, the yields of the respective natural products are given as percentage recovery or yield of the initially spiked compound quantity ( $1.0 \mathrm{mg}$ for tetracycline, cyclosporine and gentamycin and $0.5 \mathrm{mg}$ for colchicine). As expected, after the observations from the preceding qualitative comparison, gentamicin was not detected in the extracts of either extraction technique or at any pH-level. Generally, as visible in Figure 3, there were few variations among the $\mathrm{pH}$ levels within the same extraction. Generally, EtOAc extraction had a better recovery of the spiked compounds (except for gentamycin), tetracycline was recovered at about 10\% using resin and $15 \%$ using EtOAc, colchicine was recovered at levels between $50 \%$ and $70 \%$, depending on the condition, for colchicine; the resin extraction performed better. The recovery of cyclosporine was, depending on the condition, at least around 2 times higher in the EtOAc extract samples compared with the resin extracts. 
We conclude that the two extraction techniques are qualitatively comparable and that both are less suitable for polar compounds, which was expected given the chemical composition of the poly-benzyl resin. Quantitatively the EtOAc extraction showed higher yields for cyclosporine, while the other two compounds were comparable. One of the prominent advantages of EtOAc extracts is that the extracts contain fewer mediacomponents and polar metabolites, which eases downstream purification of compounds from the extracts. Furthermore, technical considerations can play a decisive role when screening larger sample collections. The presented protocol of resin extraction appears to be rather impracticable for smaller culture volumes $(>5 \mathrm{~mL})$, but on the other hand, it can be more convenient when processing larger numbers of fermentations at an intermediate scale. Since we expect many of the microbial secondary metabolites to be excreted to the media in order to exert their biological effect [4], extraction of the media seems to be reasonable. However, extraction of a $10 \mathrm{~L}$ culture using EtOAc would demand an equal volume of the organic solvent, while resin extraction would require just gram amounts of resin, but at the expense of extracting more polar molecules. It was important for us to document that the extraction methods were qualitatively comparable for the tested compounds; therefore, other technical considerations can be prioritized when deciding on one of the protocols.

\section{Conclusions}

The liquid-liquid and liquid-solid state methods are comparable when extracting the selected natural products from a microbial fermentation broth or water solutions at different $\mathrm{pH}$ levels. Both methods are less suitable for the extraction of polar metabolites. The liquid-liquid extract contains fewer media components, but this method is less suited when extracting larger cultivations due to high consumption of organic solvents.

Author Contributions: Conceptualization, E.H.H., J.H.A. and Y.K.S.; investigation, S.M.J. and Y.K.S.; writing-original draft preparation, Y.K.S.; writing—review and editing, E.H.H. and J.H.A. All authors have read and agreed to the published version of the manuscript.

Funding: Y.K.S. received funding from the Marie Skłodowska-Curie Action MarPipe, grant agreement GA 721421 H2020-MSCA-ITN-2016, of the European Union, and from UiT-The Arctic University of Norway.

Institutional Review Board Statement: Not applicable.

Informed Consent Statement: Not applicable.

Data Availability Statement: Not applicable.

Acknowledgments: Dana Elizabeth Wright (UiT-The Arctic University of Norway) is gratefully acknowledged for proofreading the manuscript.

Conflicts of Interest: The authors declare no conflict of interest.

\section{Appendix A}

Table A1. Table of calculated yields from the quantitative comparison.

\begin{tabular}{ccccccccccccc}
\hline Calc. Compound Yields in $(\mu \mathrm{g})$ & \multicolumn{4}{c}{ EtOAc Extraction } & \multicolumn{3}{c}{ Resin Extraction } \\
\hline pH & $\mathbf{4 . 0}$ & $\mathbf{5 . 0}$ & $\mathbf{6 . 0}$ & $\mathbf{7 . 0}$ & $\mathbf{8 . 5}$ & $\mathbf{4 . 0}$ & $\mathbf{5 . 0}$ & $\mathbf{6 . 0}$ & $\mathbf{7 . 0}$ & $\mathbf{8 . 5}$ \\
\hline Colchicine & 327 & 267 & 260 & 251 & 254 & 332 & 303 & 338 & 325 & 359 \\
Cyclosporine & 607 & 389 & 362 & 383 & 461 & 97 & 118 & 220 & 112 & 171 \\
Tetracycline & 134 & 88 & 78 & 106 & 44 & 70 & 77 & 69 & 58 & 59 & - \\
Gentamycin & - & - & - & - & - & - & - & - \\
\hline
\end{tabular}




\section{References}

1. Newman, D.J.; Cragg, G.M. Natural products as sources of new drugs over the nearly four decades from 01/1981 to 09/2019. J. Nat. Prod. 2020, 83, 770-803. [CrossRef]

2. Rosén, J.; Gottfries, J.; Muresan, S.; Backlund, A.; Oprea, T. Novel Chemical Space Exploration via Natural Products. J. Med. Chem. 2009, 52, 1953-1962. [CrossRef] [PubMed]

3. Ganesan, A. The impact of natural products upon modern drug discovery. Curr. Opin. Chem. Biol. 2008, 12, 306-317. [CrossRef] [PubMed]

4. Pham, J.V.; Yilma, M.A.; Feliz, A.; Majid, M.T.; Maffetone, N.; Walker, J.R.; Kim, E.; Cho, H.J.; Reynolds, J.M.; Song, M.C.; et al. A Review of the Microbial Production of Bioactive Natural Products and Biologics. Front. Microbiol. 2019, 10, 1404. [CrossRef]

5. Wagenaar, M.M. Pre-fractionated Microbial Samples-The Second Generation Natural Products Library at Wyeth. Molecules 2008, 13, 1406-1426. [CrossRef]

6. Grkovic, T.; Akee, R.K.; Thornburg, C.C.; Trinh, S.K.; Britt, J.R.; Harris, M.J.; Evans, J.R.; Kang, U.; Ensel, S.; Henrich, C.J.; et al. National Cancer Institute (NCI) Program for Natural Products Discovery: Rapid Isolation and Identification of Biologically Active Natural Products from the NCI Prefractionated Library. ACS Chem. Biol. 2020, 15, 1104-1114. [CrossRef] [PubMed]

7. Cordell, G.A.; Shin, Y.G. Finding the needle in the haystack. The dereplication of natural product extracts. Pure Appl. Chem. 1999, 71, 1089-1094. [CrossRef]

8. Ito, T.; Masubuchi, M. Dereplication of microbial extracts and related analytical technologies. J. Antibiot. 2014, 67, 353-360. [CrossRef]

9. Sashidhara, K.V.; Rosaiah, J.N. Various Dereplication Strategies Using LC-MS for Rapid Natural Product Lead Identification and Drug Discovery. Nat. Prod. Commun. 2007, 2, 193-202. [CrossRef]

10. Zou, Y.; Yin, H.; Kong, D.; Deng, Z.; Lin, S. ATrans-Acting Ketoreductase in Biosynthesis of a Symmetric Polyketide Dimer SIA7248. Chembiochem Eur. J. Chem. Biol. 2013, 14, 679-683. [CrossRef]

11. Schneider, Y.K.-H.; Hansen, K.Ø.; Isaksson, J.; Ullsten, S.; Hansen, E.H.; Hammer Andersen, J. Anti-Bacterial Effect and Cytotoxicity Assessment of Lipid 430 Isolated from Algibacter sp. Molecules 2019, 24, 3991. [CrossRef] [PubMed]

12. Schneider, Y.; Jenssen, M.; Isaksson, J.; Hansen, K.; Andersen, J.; Hansen, E. Bioactivity of Serratiochelin A, a Siderophore Isolated from a Co-Culture of Serratia sp. and Shewanella sp. Microorganisms 2020, 8, 1042. [CrossRef]

13. Hayashida-Soiza, G.; Uchida, A.; Mori, N.; Kuwahara, Y.; Ishida, Y. Purification and characterization of antibacterial substances produced by a marine bacterium Pseudoalteromonas haloplanktisstrain. J. Appl. Microbiol. 2008, 105, 1672-1677. [CrossRef]

14. Wietz, M.; Månsson, M.; Bowman, J.S.; Blom, N.S.; Ng, Y.; Gram, L. Wide Distribution of Closely Related, Antibiotic-Producing Arthrobacter Strains throughout the Arctic Ocean. Appl. Environ. Microbiol. 2012, 78, 2039-2042. [CrossRef]

15. Pinu, F.R.; Villas-Boas, S.G.; Aggio, R. Analysis of Intracellular Metabolites from Microorganisms: Quenching and Extraction Protocols. Metabolites 2017, 7, 53. [CrossRef] [PubMed]

16. Sturini, M.; Speltini, A.; Pretali, L.; Fasani, E.; Profumo, A. Solid-phase extraction and HPLC determination of fluoroquinolones in surface waters. J. Sep. Sci. 2009, 32, 3020-3028. [CrossRef] [PubMed]

17. Xu, J.; Zhu, L.-Y.; Shen, H.; Zhang, H.-M.; Jia, X.-B.; Yan, R.; Li, S.-L.; Xu, H.-X. A critical view on spike recovery for accuracy evaluation of analytical method for medicinal herbs. J. Pharm. Biomed. Anal. 2012, 62, 210-215. [CrossRef]

18. Grosso, C.; Valentão, P.; Ferreres, F.; Andrade, P.B. Alternative and Efficient Extraction Methods for Marine-Derived Compounds. Mar. Drugs 2015, 13, 3182-3230. [CrossRef] [PubMed]

19. Baldino, L.; Scognamiglio, M.; Reverchon, E. Supercritical fluid technologies applied to the extraction of compounds of industrial interest from Cannabis sativa L. and to their pharmaceutical formulations: A review. J. Supercrit. Fluids 2020, 165, 104960. [CrossRef]

20. Pintać, D.; Majkić, T.; Torovic, L.; Orčić, D.; Beara, I.; Simin, N.; Mimica-Dukić, N.; Lesjak, M. Solvent selection for efficient extraction of bioactive compounds from grape pomace. Ind. Crop. Prod. 2018, 111, 379-390. [CrossRef]

21. Hedrich, S.; Schippers, A. Distribution of Acidophilic Microorganisms in Natural and Man-made Acidic Environments. Curr. Issues Mol. Biol. 2021, 40, 25-48. [CrossRef] [PubMed]

22. Ding, Z.-G.; Li, M.-G.; Zhao, J.-Y.; Ren, J.; Huang, R.; Xie, M.-J.; Cui, X.-L.; Zhu, H.-J.; Wen, M.-L. Naphthospironone A: An Unprecedented and Highly Functionalized Polycyclic Metabolite from an Alkaline Mine Waste Extremophile. Chem.-A Eur. J. 2010, 16, 3902-3905. [CrossRef] [PubMed] 